\title{
Gastric hepatoid adenocarcinoma resulting in a spontaneous gastric perforation: a case report and review of the literature
}

\author{
Junichi Yoshizawa ${ }^{1,3^{*}}$, Satoshi Ishizone ${ }^{1}$, Meguru Ikeyama² and Jun Nakayama ${ }^{2}$
}

\begin{abstract}
Background: Gastric hepatoid adenocarcinoma (GHAC) is an atypical form of gastric cancer (GC) that has similar tissue morphology to hepatocellular carcinoma and frequently produces alpha-fetoprotein. We present an exceedingly rare case of GHAC resulting in a spontaneous gastric perforation.

Case presentation: A 61-year-old man presented at our institution complaining of abdominal and back pain. A computed tomography scan revealed a spontaneous gastric perforation with a solitary liver tumor and lymph node swelling. Following a diagnosis of advanced-stage GC with a gastric perforation, perforative peritonitis, multiple lymph node metastases, and a solitary metastasis of the lateral segment of the liver, the patient underwent distal gastrectomy. Histopathology of the resected specimen revealed that the tumor cells were arranged in a hepatoid pattern. On immunohistochemical staining, the tumor cells were positive for alpha-fetoprotein and Sal-like protein 4. Thus, the patient was diagnosed with GHAC. Hepatic resection of the solitary liver metastasis was performed. However, recurrence occurred and the patient achieved complete response following tegafur/gimeracil/oteracil-based chemotherapy.
\end{abstract}

Conclusions: GHAC is a highly malignant histological subtype of GC. We reported on an extremely rare case of GHAC resulting in a spontaneous gastric perforation and reviewed the literature, including epidemiological data, treatment regimens, and the association between GHAC and alpha-fetoprotein-producing GC.

Keywords: Alpha-fetoprotein, Case report, Gastric cancer, Gastric perforation, Hepatoid adenocarcinoma

\section{Background}

Hepatoid adenocarcinoma is a malignant cancer manifesting outside the liver that most frequently arises in the stomach, with gastric hepatoid adenocarcinoma (GHAC) accounting for $63 \%$ of cases. Hepatoid adenocarcinoma also arises in the ovaries (10\%), lungs (5\%), bladder (4\%), pancreas (4\%), and uterus (4\%) [1]. GHAC is a rare form of gastric cancer (GC) that accounts for $\leq 1 \%$ of all GCs [2-4]. GHAC has been recognized in approximately 500 cases to date, mainly in case reports and clinical or pathological analyses that were identified from literature searches of the PubMed database using the search terms:

\footnotetext{
*Correspondence: ciel001100@gmail.com

'Department of Surgery, North Alps Medical Center Azumi Hospital, 3207-1

Ikeda, Ikeda-machi, Kitaazumi-gun, Nagano Prefecture 399-8695, Japan

${ }^{3}$ Present Address: Suwa Red Cross Hospital, 5-11-50 Kogandori, Suwa-shi,

Nagano Prefecture 392-8510, Japan

Full list of author information is available at the end of the article
}

\begin{abstract}
"hepatoid adenocarcinoma of the stomach" AND "gastric hepatoid adenocarcinoma" [4, 5]. Among the different GC subtypes, GHAC has comparable histology and functionality to stem cell differentiation, it has pathologically similar tissue morphology to hepatocellular carcinoma (HCC), and it frequently expresses alpha-fetoprotein (AFP) on immunohistochemistry [6, 7]. GHACs progress rapidly, with the majority of patients presenting with lymph node (LN) or liver metastases. The risk of recurrence in GHAC patients is high, even after radical resection. Currently, no standard chemotherapy regimen has been established [8]. For these reasons, the prognosis of patients with GHAC remains especially poor. Liu et al. [2] reported that the 1-, 3 -, and 5-year survival rates of GHAC versus non-GHAC patients were $30 \%, 13 \%$, and $9 \%$, and $96 \%, 61 \%$, and $44 \%$, respectively. GHAC patients had a statistically significant poorer prognosis than non-GHAC patients [2]. GHAC has no specific symptoms with many common symptoms
\end{abstract}


of GC having been observed (e.g., general fatigue, reduced appetite, gastric distention, epigastric pain, anemia, and melena) [8]. We present an exceedingly rare case of GHAC resulting in a spontaneous gastric perforation and review the literature, including epidemiological data, treatment regimens, and the association between GHAC and AFP-producing GC.

\section{Case presentation}

A 61-year-old man experienced upper abdominal and lower left back pain 1 month and 1 week prior to examination, respectively. He was referred to our hospital after the pain had worsened. The patient experienced spontaneous lumbar and epigastric pain with muscular defense of the epigastrium and accompanying tenderness. Blood test results indicated a white blood cell count of $12,430 / \mu \mathrm{L}$, a $\mathrm{C}$-reactive protein level of $0.6 \mathrm{mg} / \mathrm{dL}$, and mild but increasing inflammation. No abnormal findings were reported from the other blood counts, biochemical examinations, and coagulation tests.

Abdominal contrast-enhanced computed tomography (CT) revealed disruption and thickening of the anterior wall of the gastric antrum. CT also revealed the presence of ascites and free air at the ventral side of the stomach and on the surface of the liver (Fig. 1a). Several enlarged LNs (maximum diameter, $30 \mathrm{~mm}$ ) were identified along the greater gastric curve and a low enhanced lesion (measuring $30 \times 25 \mathrm{~mm}$ ) was detected on the lateral segment of the liver (Fig. 1b). A diagnosis of advanced-stage GC resulting in a spontaneous gastric perforation, with perforative peritonitis, multiple LN metastases, and a solitary liver metastasis was made and an emergency laparotomy was performed on the same day. A moderate amount of turbid ascites was observed in the abdominal cavity during laparotomy. A 7-mm perforation of the gastric antrum was detected, along with marked thickening of the gastric wall and coarse neoplastic tumors that were attached to the gastric wall (Fig. 2). The tumors were exposed on the serosal surface along the gastric perforation. The patient was diagnosed with advancedstage GC resulting in a spontaneous gastric perforation. No peritoneal dissemination was observed. Preoperative $\mathrm{CT}$ also revealed the presence of metastases in several enlarged LNs along the greater gastric curve, as well as, a number of hardened regions in the lateral segment of the liver. A distal gastrectomy with radical lymphadenectomy and cholecystectomy was performed. Reconstructive surgery was achieved using Billroth II anastomosis. No postoperative complications occurred and the patient was discharged.

Gross findings of the resected specimen included an ulcerative and infiltrative (type 3 ) tumor (approximately $100 \times 50 \mathrm{~mm}$ ) with an infiltrative serosal surface and 7$\mathrm{mm}$ puncture sites (Fig. 3). Microscopic findings revealed that the tumor was comprised of a homogeneous proliferation of polygonal tumor cells with abundant, eosinophilic, and clear cytoplasm. Numerous mitoses were also detected. The tumor cells exhibited solid or thicktrabecular patterns with scanty stroma containing blood vessels that resembled $\mathrm{HCC}$ and expansive invasion into the gastric wall (Fig. 4). Features of enteroblastic differentiation and Schiller-Duval bodies were absent.

Immunohistochemical staining was performed to characterize the tumor cells (Table 1). The tumor cells stained positive for AFP and Sal-like protein 4 (SALL4), but were negative for carcinoembryonic antigen, synaptophysin, chromogranin $\mathrm{A}$, and neural cell adhesion molecule (Fig. 5a-f). According to these findings, the patient was diagnosed with GHAC. No tumor cells were observed during the cytological examination of ascites. LN metastases were detected in 6 LNs (20\%) and a solitary metastasis was detected on the lateral segment of the liver. Subsequently, the patient was classified as having a Stage IV (T4aN2M1) GHAC. Moderate venous and lymphatic infiltration was observed. Postoperative blood biochemical analysis revealed an elevated AFP level $(487.4 \mathrm{ng} / \mathrm{mL})$, which supported a diagnosis of GHAC.

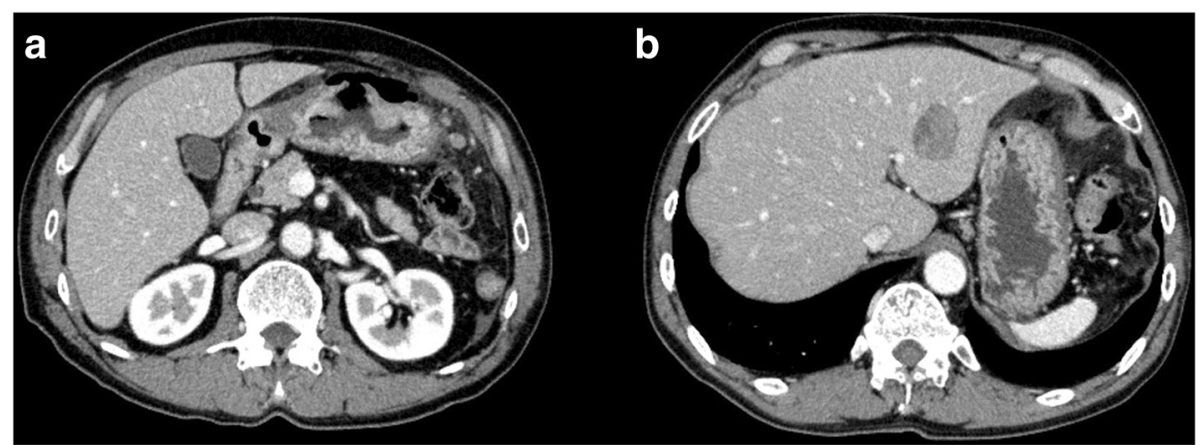

Fig. 1 Imaging findings. Abdominal contrast-enhanced computed tomography revealed disruption and thickening of the anterior wall of the gastric antrum with free air (a) and a low enhanced lesion (30 $\mathrm{mm}$ in diameter) on the lateral segment of the liver (b) 


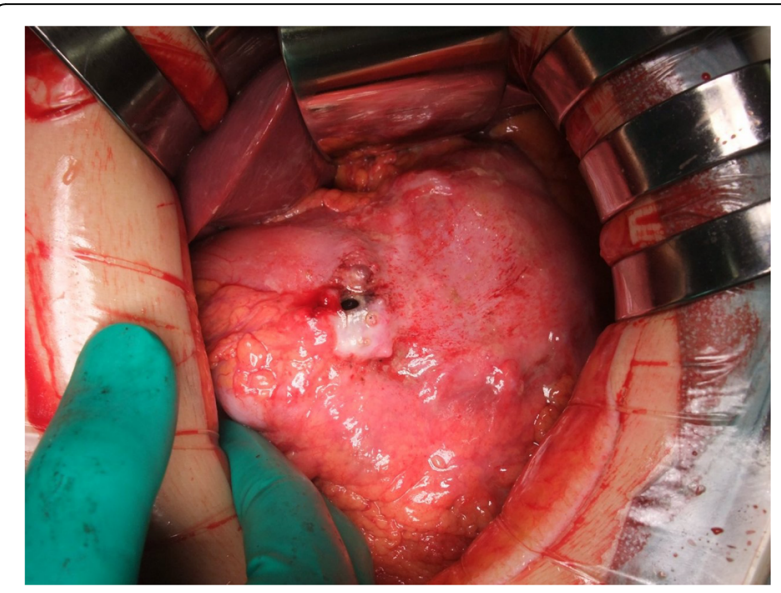

Fig. 2 Intraoperative findings. A 7-mm perforation of the anterior wall of the gastric antrum with serous exposure of bulky tumors and opacity ascites

The patient was followed-up for 11 weeks postgastrectomy to monitor the occurrence of new LN or liver metastases. Because no new lesions were detected, resection of the lateral segment of the liver was performed, at another hospital, 13 weeks after the initial operation. The patient was confirmed from histopathological examinations as having GHAC with a solitary liver metastasis. An elevated serum AFP level (1214.9 $\mathrm{ng} / \mathrm{mL})$ was recorded 6 weeks after hepatic resection and recurrence of LN metastasis was detected on CT. Combination chemotherapy with tegafur/gimeracil/oteracil (S-1) was administered at a dose of $120 \mathrm{mg} /$ day for 2 weeks with a 1-week rest. Three months later, serum AFP levels had normalized and shrinkage of the resected LN was observed. Elevated serum AFP levels were not detected 15 months post-

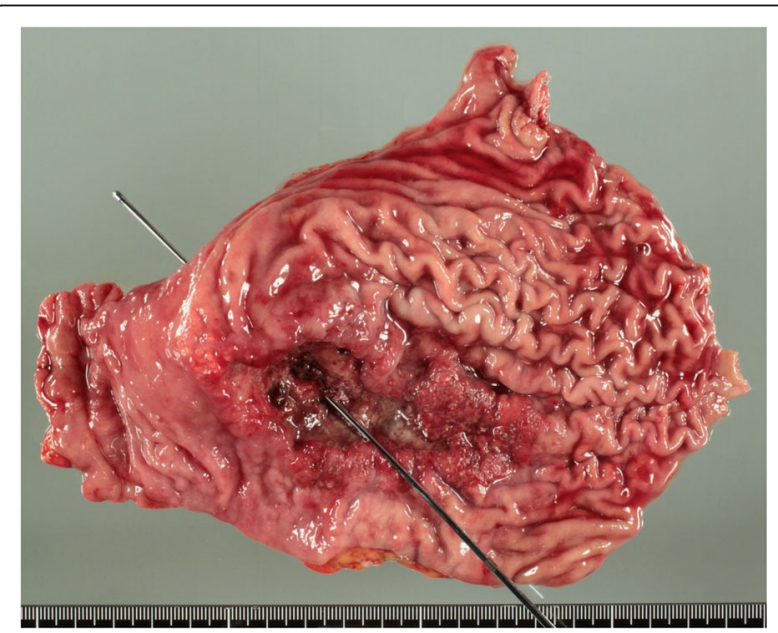

Fig. 3 Gross findings. Ulcerative and infiltrative (type 3) tumor of the gastric antrum (approximately $100 \times 50 \mathrm{~mm}$ ) with an infiltrative serosal surface and central perforation of the gastric cancer recurrence and complete response of the $\mathrm{LN}$ metastasis was achieved.

\section{Discussion and conclusions}

Boureille et al. [9] first reported on the use of elevated serum AFP levels as a biomarker for GC in 1970. In 1977, Okita et al. [10] verified the expression of AFP in $\mathrm{GC}$, through immunohistochemistry, and established the concept of "AFP-producing GC". AFP-producing GC does not reflect a diagnosis of a specific histological subtype, but rather describes a group of tumor histologies that have the capacity to produce AFP. These include gastric hepatoid, enteroblastic, and yolk sac tumors $[11,12]$. It should be noted that GC with enteroblastic differentiation is distinguishable from gastroblastoma, which is a neoplasm that is rarely observed in children or young adults and is considered a low-grade malignancy $[13,14]$.

In 1985, Ishikura et al. [6] introduced the concept of GHAC after conducting an investigation of AFPproducing $\mathrm{GC}$ cases with morphological features mimicking HCC. However, since a proportion of GHAC patients do not express AFP, Nagai et al. [5] suggested that GHAC should be diagnosed based on its histological characteristics, irrespective of its capacity to produce AFP. Since AFP production has been observed in the fetal liver, HCCs, and GHACs, GHAC is considered to represent a gastric carcinoma with hepatic differentiation and morphological similarity to hepatic cells [15].

Inagawa et al. [8] investigated 85 GHAC patients (mean age, 63.5 [range, 44-87] years with a male-tofemale ratio of 2.3:1) and reported that GHAC had occurred relatively more frequently in middle-aged men than in elderly men. GHAC originated in the gastric antrum in $60 \%$ of patients. Only $13 \%$ of patients were diagnosed with early-stage GHAC. Gross findings suggested the presence of type 2 or type 3 ulcerative lesions in $29(62 \%)$ of 47 cases. The majority of patients presented with $\mathrm{LN}$ and liver metastases. These findings were consistent with the findings of our case.

GHAC is not associated with specific symptoms and many common symptoms of GC are observed (e.g., general fatigue, reduced appetite, gastric distention, epigastric pain, anemia, and melena) [8]. In our case, the patient developed upper abdominal and back pain, and a spontaneous gastric perforation was detected on CT.

Histopathologically, GHAC resembles HCC. The tumor cells grow, proliferate, and invade surrounding tissues with significant accompanying venous infiltration $[11,16]$. GHACs are frequently associated with highly differentiated papillary adenocarcinomas. It is thought that GHACs may arise from these tumors through hepatic differentiation [15-17]. However, such a 


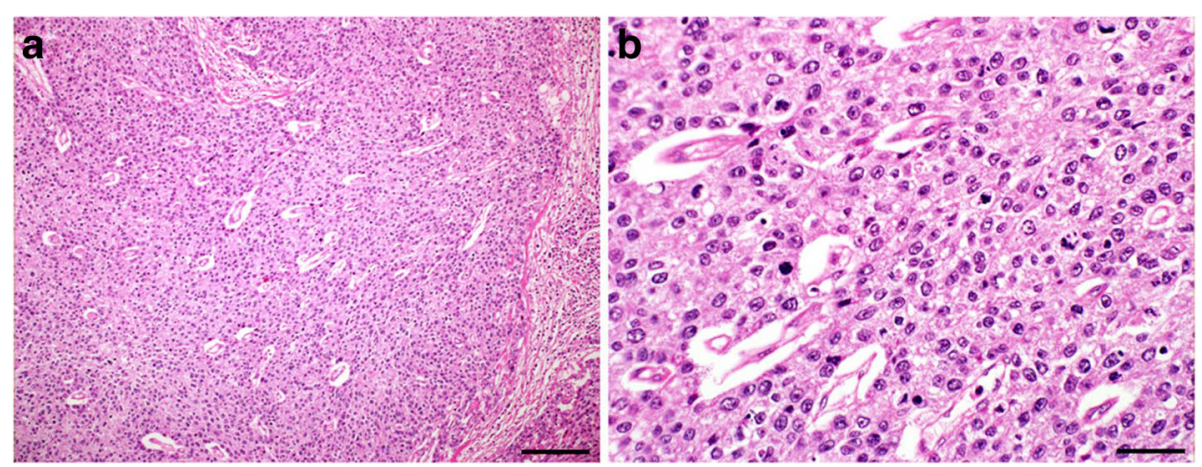

Fig. 4 Histopathological findings. Hematoxylin and eosin staining of proliferating tumor cells with solid or thick-trabecular patterns mimicking hepatocellular carcinoma. The scale bars in (a) and (b) indicate $200 \mu \mathrm{m}$ and $50 \mu \mathrm{m}$, respectively

differentiated adenocarcinoma was not detected in the present case.

AFP and glypican-3 are oncofetal proteins that are produced by the fetal liver, yolk sac tumors, hepatoblastomas, and HCCs [18]. Because these proteins are also frequently expressed in enteroblastic gastric tumors, as well as GHACs, each tumor is classified as GC exhibiting fetal differentiation. SALL4 expression has been observed in the neofetal stomach, primitive germ cell tumors, enteroblastic adenocarcinomas, yolk sac tumors, and GHACs [19]. Since AFP expression is often negative in GHACs, whereas glypican-3 and SALL4 expression are usually positive, glypican-3 and SALL4 are considered potentially more useful clinical biomarkers of GHAC than AFP. Moreover, SALL4 is not expressed in normal liver tissue or HCCs. Therefore, SALL4 expression may be useful for distinguishing GHACs from HCCs [5, 18-20]. In our case, both AFP and SALL4 expression, as well as, morphological features mimicking $\mathrm{HCC}$ meant we were able to diagnose the tumor as a GHAC.

Although GC resulting in a gastric perforation is rare (accounting for $<1 \%$ of all GC cases [21-24]), the possibility of GC should be considered when a diagnosis of a gastric perforation is confirmed. The diagnosis of a gastric perforation in GC patients, either before or during surgery, is not necessarily straightforward, with diagnoses frequently made based on ulcer size, the extent of sclerosis, the presence of LN and liver metastases, and gastric dissemination. In our case, coarse neoplastic tumors and serosal invasion were observed, along with suspected LN and liver metastases. Accordingly, the patient was confirmed intraoperatively as having a GC-induced gastric perforation. The treatment strategy included primary gastric resection and repair surgery, which were primarily selected based on the presence of preoperative shock, the extent of peritonitis, neoplasm curability, and the patient's comorbidities [25]. Primary gastric resection involves gastrectomy with radical lymphadenectomy and palliative gastrectomy. Repair surgery is usually performed to close the perforation using an omental patch. Following repair surgery, patients frequently undergo a secondary gastric resection or are administered chemotherapy for GC. In our case, gastrectomy with LN dissection was performed, because we had diagnosed a GC-induced perforation without peritoneal dissemination or distant metastases (except for a solitary liver metastasis) and the patient's general condition was stable without comorbidities. Since the patient suffered from severe perioperative

Table 1 Antibodies used for immunohistochemical analysis

\begin{tabular}{|c|c|c|c|c|c|}
\hline Antibody & Clone & Supplier & Supplier location & Detection kit & Supplier \\
\hline $\begin{array}{l}\text { Carcinoembryonic antigen } \\
\text { (CEA) }\end{array}$ & $\begin{array}{l}\text { TF3H8-1 (mouse, } \\
\text { monoclonal) }\end{array}$ & $\begin{array}{l}\text { Ventana Medical } \\
\text { Systems }\end{array}$ & Tucson, AZ, USA & Ventana ultraView DAB & $\begin{array}{l}\text { Ventana Medical } \\
\text { Systems }\end{array}$ \\
\hline Synaptophysin & $\begin{array}{l}\text { MRQ-40 (rabbit, } \\
\text { monoclonal) }\end{array}$ & $\begin{array}{l}\text { Ventana Medical } \\
\text { Systems }\end{array}$ & Tucson, AZ, USA & Ventana ultraView DAB & $\begin{array}{l}\text { Ventana Medical } \\
\text { Systems }\end{array}$ \\
\hline Chromogranin A & $\begin{array}{l}\text { LK2H10 (mouse, } \\
\text { monoclonal) }\end{array}$ & $\begin{array}{l}\text { Ventana Medical } \\
\text { Systems }\end{array}$ & Tucson, AZ, USA & Ventana ultraView DAB & $\begin{array}{l}\text { Ventana Medical } \\
\text { Systems }\end{array}$ \\
\hline $\begin{array}{l}\text { Neural cell adhesion } \\
\text { molecule (NCAM) }\end{array}$ & $\begin{array}{l}123 C 3 \text { (mouse, } \\
\text { monoclonal) }\end{array}$ & Monosan & Uden, The Netherlands & $\begin{array}{l}\text { Histofine Simple Stain } \\
\text { MAX-PO(M) }\end{array}$ & Nichirei Biosciences \\
\hline Alpha-fetoprotein & A0008 (rabbit, polyclonal) & DAKO & Glostrup, Denmark & $\begin{array}{l}\text { Histofine Simple Stain } \\
\text { MAX-PO(R) }\end{array}$ & Nichirei Biosciences \\
\hline Sal-like protein 4 (SALL4) & 6E3 (mouse monoclonal) & Abcam & Cambridge, UK & $\begin{array}{l}\text { Histofine Simple Stain } \\
\text { MAX-PO(M) }\end{array}$ & Nichirei Biosciences \\
\hline
\end{tabular}




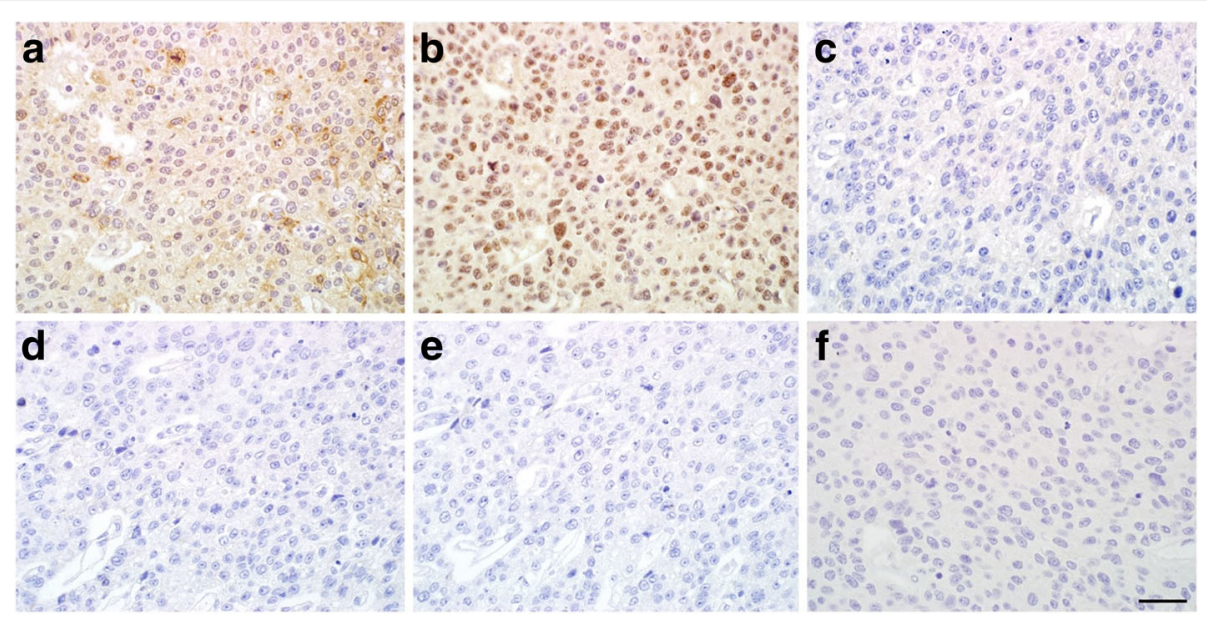

Fig. 5 Immunohistochemical analysis. Tumor cells stained positive for (a) alpha-fetoprotein and (b) Sal-like protein 4, but were negative for (c) carcinoembryonic antigen, (d) synaptophysin, (e) chromogranin A, and (f) neural cell adhesion molecule. The scale bar indicates $50 \mu \mathrm{m}$

peritonitis, we anticipated that a high degree of peritoneal adhesion would arise from severe peritoneal inflammation. Therefore, we believed that if, during the initial operation, we had performed gastrectomy or repair surgery without lymphadenectomy, then it would have been difficult to perform surgery with lymphadenectomy at a later date. To the best of our knowledge, the only previous case of GHAC involving a gastric perforation has been reported in a pediatric patient [26]. Therefore, our case is the first involving an adult patient.

Currently, a standard treatment regimen for GHAC is lacking and treatments designed for GC are being administered. In instances where GHAC is accompanied by liver metastases, radical resection should be considered, provided the number of liver metastases is limited and liver resection is achievable [20,27]. However, in a significant proportion of GHAC patients, early recurrence occurs, even when preventative LN dissection and radical resection have been performed [3]. In contrast, palliative surgery may be conducted in instances where distant metastases are present and radical resection is not achievable. In our case, since the liver metastasis was solitary, liver resection was performed and no metastases to the LNs or other organs were observed during the 11-week follow-up period. Unfortunately, however, LN metastases were detected shortly thereafter.

Although the cause is unknown, $\mathrm{LN}$ and liver metastases could easily develop given the extent of $\mathrm{LN}$ and venous invasion accompanying GHACs. GHACs also progress relatively rapidly. Therefore, GHAC is considered to be highly malignant [28]. Nagai et al. [5] reported that GHAC is associated with a poor prognosis compared to other forms of AFP-producing GC, which typically have a poorer prognosis than classical GC. Beak et al. [3] reported mean survival periods of 28.0 and 8.0 months for
Stage I-III and Stage IV patients, respectively. Recently, $\mathrm{Qu}$ et al. [4] reviewed 95 GHAC cases from China and reported a 3-year survival rate of $7.4 \%$ and a median survival time of 10 months. The high malignancy of GHAC is associated with a high degree of tumor vascularization and rapid cell proliferation. Koide et al. [29] reported elevated levels of the Ki-67 labeling index and vascular endothelial growth factor expression in AFP-producing versus non-AFP-producing GC patients. Moreover, Inagawa et al. [8] demonstrated elevated levels of Ki-67 expression in GHAC tumors that was associated with tumor cell proliferation, relatively weak apoptosis, and rich neovascularization.

Treatment for GHAC patients with unresectable metastases, including S-1 monotherapy and paclitaxel/ cisplatin-based, oxaliplatin/capecitabine-based, and fluoropyrimidine/platinum-based chemotherapy has been effective in some instances [3, 30]. However, to date, no antitumor drug has been established as the gold standard of treatment. In our case, shrinkage of the recurrent LN metastases and normalized serum AFP levels were observed following administration of S-1 monotherapy and a steady effect with respect to GHAC was achieved.

To improve the prognoses of patients with GHAC and AFP-producing GC, as well as, diagnostic accuracy and incorporation of radical resections, multimodality therapy (including supplementary chemotherapy) and the establishment of a standard chemotherapy regimen are required to address instances of recurrent tumors. Even when radical resections are performed, recurrences and additional metastases frequently occur. Therefore, closer monitoring is needed.

We describe a rare case of GHAC resulting in a spontaneous gastric perforation. In our patient, the GHAC was accompanied by multiple LN metastases and a solitary 
liver metastasis. Distal gastrectomy and lateral liver lobe resection were performed. However, the patient developed postoperative recurrence of LN metastasis. S-1 monotherapy was administered and complete response of the $\mathrm{LN}$ metastases was achieved.

GHACs progress rapidly and are characterized by a high frequency of LN and liver metastases. Therefore, GHACs are considered highly malignant tumors with poor prognoses. We anticipate that both clinical and basic research will continue to advance with the accumulation of future cases.

\section{Abbreviations}

AFP: Alpha-fetoprotein; CT: Computed tomography; GC: Gastric cancer; GHAC: Gastric hepatoid adenocarcinoma; HCC: Hepatocellular carcinoma; LN: Lymph node; S-1: Tegafur/gimeracil/oteracil; SALL4: Sal-like protein 4

\section{Acknowledgements}

We wish to thank Mr. Noriyasu Kobayashi (North Alps Medical Center Azumi Hospital, Nagano, Japan) for his technical assistance in performing the pathological analyses.

\section{Funding}

None.

\section{Availability of data and materials}

The datasets supporting the conclusions of this article are included within the article.

\section{Authors' contributions}

$\mathrm{JY}$ and SI performed the gastrectomy and MI and JN performed the pathological analyses. JY contributed to the conception and design of the Case Report and drafted the manuscript. SI followed-up the patient and conducted the chemotherapy treatment. All authors have read and approved the final version of this manuscript.

\section{Competing interests}

The authors declare that they have no competing interests.

\section{Consent for publication}

Written informed consent was obtained from the patient for publication of this Case Report and any accompanying images. A copy of the written consent is available for review by the Editor of this journal.

\section{Ethics approval and consent to participate}

Not applicable.

\section{Publisher's Note}

Springer Nature remains neutral with regard to jurisdictional claims in published maps and institutional affiliations.

\section{Author details \\ 'Department of Surgery, North Alps Medical Center Azumi Hospital, 3207-1 Ikeda, Ikeda-machi, Kitaazumi-gun, Nagano Prefecture 399-8695, Japan. ${ }^{2}$ Department of Molecular Pathology, Shinshu University Graduate School of Medicine, 3-1-1 Asahi, Matsumoto, Nagano Prefecture 390-8621, Japan. ${ }^{3}$ Present Address: Suwa Red Cross Hospital, 5-11-50 Kogandori, Suwa-shi, Nagano Prefecture 392-8510, Japan.}

Received: 23 November 2016 Accepted: 15 May 2017

Published online: 25 May 2017

\section{References}

1. Metzgeroth G, Ströbel P, Baumbusch T, Reiter A, Hastka J. Hepatoid adenocarcinoma - review of the literature illustrated by a rare case originating in the peritoneal cavity. Onkologie. 2010;33:263-9.
2. Liu X, Cheng Y, Sheng W, Lu H, Xu X, Xu Y, et al. Analysis of clinicopathologic features and prognostic factors in hepatoid adenocarcinoma of the stomach. Am J Surg Pathol. 2010;34:1465-71.

3. Baek SK, Han SW, Oh DY, Im SA, Kim TY, Bang YJ. Clinicopathologic characteristics and treatment outcomes of hepatoid adenocarcinoma of the stomach, a rare but unique subtype of gastric cancer. BMC Gastroenterol. 2011;11:56.

4. Qu BG, Bi WM, Qu BT, Qu T, Han XH, Wang H, et al. PRISMA-compliant article: clinical characteristics and factors influencing prognosis of patients with hepatoid adenocarcinoma of the stomach in china. Medicine (Baltimore). 2016; doi:10.1097/MD.0000000000003399.

5. Nagai E, Ueyama T, Yao T, Tsuneyoshi M. Hepatoid adenocarcinoma of the stomach. A clinicopathologic and immunohistochemical analysis. Cancer. 1993;72:1827-35.

6. Ishikura H, Fukasawa Y, Ogasawara K, Natori T, Tsukada Y, Aizawa M. An AFP-producing gastric carcinoma with features of hepatic differentiation. A case report. Cancer. 1985;56:840-8.

7. Ishikura H, Kishimoto T, Andachi H, Kakuta Y, Yoshiki T. Gastrointestinal hepatoid adenocarcinoma: venous permeation and mimicry of hepatocellular carcinoma, a report of four cases. Histopathology. 1997;31:47-54.

8. Inagawa S, Shimazaki J, Hori M, Yoshimi F, Adachi S, Kawamoto T, et al. Hepatoid adenocarcinoma of the stomach. Gastric Cancer. 2001:4:43-52.

9. Bourreille J, Metayer P, Sauger F, Matray F, Fondimare A. Existence of alpha feto protein during gastric-origin secondary cancer of the liver. Presse Med. 1970;78:1277-8.

10. Okita K, Noda K, Kodama T, Takenami T, Fukumoto Y, Fujii R, et al. Carcinofetal proteins and gastric cancer: the site of alpha-fetoprotein synthesis in gastric cancer. Gastroenterol Jpn. 1977;12:400-6.

11. Kumashiro Y, Yao T, Aishima S, Hirahashi M, Nishiyama K, Yamada T, et al. Hepatoid adenocarcinoma of the stomach: histogenesis and progression in association with intestinal phenotype. Hum Pathol. 2007;38:857-63.

12. Motoyama T, Aizawa K, Watanabe H, Fukase M, Saito K. Alpha-fetoprotein producing gastric carcinomas: a comparative study of three different subtypes. Acta Pathol Jpn. 1993;43:654-61

13. Wey EA, Britton AJ, Sferra JJ, Kasunic T, Pepe LR, Appelman HD. Gastroblastoma in a 28-year-old man with nodal metastasis: proof of the malignant potential. Arch Pathol Lab Med. 2012;136:961-4.

14. Ma Y, Zheng J, Zhu H, Dong K, Zheng S, Xiao X, et al. Gastroblastoma in a 12-year-old Chinese boy. Int J Clin Exp Pathol. 2014;7:3380-4.

15. Ishikura $H$, Kirimoto $K$, Shamoto M, Miyamoto $Y$, Yamagiwa $H$, Itoh T, et al. Hepatoid adenocarcinomas of the stomach. An analysis of seven cases. Cancer. 1986;58:119-26.

16. Kinjo T, Taniguchi H, Kushima R, Sekine S, Oda I, Saka M, et al. Histologic and immunohistochemical analyses of a-fetoprotein-producing cancer of the stomach. Am J Surg Pathol. 2012;36:56-65.

17. Kishimoto T, Nagai Y, Kato K, Ozaki D, Ishikura H. Hepatoid adenocarcinoma: a new clinicopathological entity and the hypotheses on carcinogenesis. Med Electron Microsc. 2000;33:57-63.

18. Ushiku T, Uozaki H, Shinozaki A, Ota S, Matsuzaka K, Nomura S, et al Glypican 3-expressing gastric carcinoma: distinct subgroup unifying hepatoid, clear-cell, and alpha-fetoprotein-producing gastric carcinomas. Cancer Sci. 2009;100:626-32.

19. Ushiku T, Shinozaki A, Shibahara J, Iwasaki Y, Tateishi Y, Funata N, et al. SALL4 represents fetal gut differentiation of gastric cancer, and is diagnostically useful in distinguishing hepatoid gastric carcinoma from hepatocellular carcinoma. Am J Surg Pathol. 2010;34:533-40.

20. Sun W, Liu Y, Shou D, Sun Q, Shi J, Chen L, et al. AFP (alpha fetoprotein): who are you in gastrology? Cancer Lett. 2015;357:43-6.

21. Cortese AF, Zahn D, Cornell GN. Perforation in gastric malignancy. J Surg Oncol. 1972:4:190-206.

22. Adachi Y, Mori M, Maehara Y, Matsumata T, Okudaira Y, Sugimachi K. Surgical results of perforated gastric carcinoma: an analysis of 155 Japanese patients. Am J Gastroenterol. 1997;92:516-8.

23. Kasakura Y, Ajani JA, Mochizuki F, Morishita Y, Fujii M, Takayama T. Outcomes after emergency surgery for gastric perforation or severe bleeding in patients with gastric cancer. J Surg Oncol. 2002;80:181-5.

24. Tsujimoto H, Hiraki S, Sakamoto N, Yaguchi Y, Horio T, Kumano I, et al. Outcome after emergency surgery in patients with a free perforation caused by gastric cancer. Exp Ther Med. 2010;1:199-203.

25. Roviello F, Rossi S, Marrelli D, De Manzoni G, Pedrazzani C, Morgagni P, et al. Perforated gastric carcinoma: a report of 10 cases and review of the literature. World J Surg Oncol. 2006;4:19. 
26. Emir S, Karakurt N, Karakuş E, Şenel E, Kırsaçlıoğlu C, Demir HA, et al. Alpha-fetoprotein-producing hepatoid gastric adenocarcinoma in a child presenting with spontaneous gastric perforation. Turk J Pediatr. 2014;56:88-91.

27. Xiao C, Wu F, Jiang H, Teng L, Song F, Wang Q, et al. Hepatoid adenocarcinoma of the stomach: nine case reports and treatment outcomes. Oncol Lett. 2015;10:1605-9.

28. Hirajima S, Komatsu S, Ichikawa D, Kubota T, Okamoto K, Shiozaki A, et al. Liver metastasis is the only independent prognostic factor in AFP-producing gastric cancer. World J Gastroenterol. 2013;19:6055-61.

29. Koide N, Nishio A, Igarashi J, Kajikawa S, Adachi W, Amano J. Alphafetoprotein-producing gastric cancer: histochemical analysis of cell proliferation, apoptosis, and angiogenesis. Am J Gastroenterol. 1999;94:1658-63.

30. Shen Z, Liu X, Lu B, Ye M. Hepatoid adenocarcinoma of the stomach: a case report of a rare type of gastric cancer. Oncol Lett. 2016;11:1077-80.

Submit your next manuscript to BioMed Central and we will help you at every step:

- We accept pre-submission inquiries

- Our selector tool helps you to find the most relevant journal

- We provide round the clock customer support

- Convenient online submission

- Thorough peer review

- Inclusion in PubMed and all major indexing services

- Maximum visibility for your research

Submit your manuscript at www.biomedcentral.com/submit
C) Biomed Central 